\title{
An Analysis on Transforming Developments in Electronic Hospital Management \& Hospital Information Systems
}

\begin{abstract}
Today's global environment service delivery models have strongly impacted by the fast growth in Information \& Communication Technology (ICT) and Internet power. E-Hospital Management systems provides the advantages of enhanced administration \& control, streamlined operations, superior patient care, strict cost control and improved profitability. Globally accepted health care systems need to comply with Healthcare Insurance Portability and Accountability Act (HIPAA) standards of the US and that has become the mode of the Healthcare industry when it comes to patient information privacy and medical records management. The aim of the study is to understand the performance indicator of Hospital Information Systems, HIS components, summarizing the latest agreed standards \& protocols like Health Level Seven (HL7), standards for mutual message exchange, etc. The study is qualitative and descriptive in nature and most of the data is based on secondary sources of survey data. To get clear idea on E-Hospital Management, Hospital information system, previous existing survey data and specific successful case studies of HIS are considered in the study. With so many customized versions of E-Hospital Management Solutions (E-HMS) and Hospital Information Systems (HIS) available in the market, a generic module wise version of E-Hospital management system is charted out to give a clear understanding for researchers and industry experts. From the specific case studies, the success factors and challenges faced in successful E-HMS implementation are highlighted. Some of the mandatory standards like HIPAA are discussed in detail for clarity on Healthcare system implementation requirements.
\end{abstract}

\section{Introduction}

Hospital Information systems are popular to handle expanding population needs furthermore helps the rehearsing specialists and hospital administration and bolster staff with convenient administration and accuracy. There are fluctuated measurements accessible to survey the execution of services like hospital industry and the effective usage and utilization of Hospital information system shapes a pivotal part. Hospital information systems are accessible in the product market which as a rule should be altered and sometimes HIS should be created as a modified programming taking into account particular hospital prerequisites (client necessities). The paper takes a gander at surveying and distinguishing the key segments of E-HMS as its needs and management changes over the globe. Additionally distinguishing proof of the key execution pointers of E-HMS/HIS is likewise endeavored from a benchmarking viewpoint.

Hospital Services are needed on an emergency and everyday schedule and HIS assumes a critical part. Hospital services are

\section{Journal of \\ Clinical Trials \& Patenting}

\author{
Mohammad Chand Jamali* \\ Department of Health \& Medical Sciences, Khawarizmi International \\ College, United Arab Emirates \\ *Address for Correspondence: \\ Mohammad Chand Jamali, Department of Health \& Medical Sciences, \\ Khawarizmi International College, 25669, Abu Dhabi, United Arab \\ Emirates, E-mail: mjamali68@gmail.com \\ Submission: 06 December, 2016 \\ Accepted: 07 January, 2017 \\ Published: 11 January, 2017 \\ Copyright: $\odot 2017$ Jamali MC. This is an open access article distributed \\ under the Creative Commons Attribution License, which permits \\ unrestricted use, distribution, and reproduction in any medium, provided \\ the original work is properly cited.
}

client and society delicate and the nature of HIS and administration of hospital staff needs to be exact and of most noteworthy norms. Today's hello there tech Hospital services are transcendently given by private players in the business sector at expanded expenses regardless of minimal effort rivalry by open area hospitals. OECD report (2012) finds that endeavors to control costs by administrative means, for example, diminishing charges paid to healthcare suppliers and proportioning client access, have regularly just been incidentally fruitful.

E-Hospital Management System gives the advantages of streamlined operations, improved organization \& control, predominant patient care, strict expense control and enhanced gainfulness. Because of business and lawful requests like the Healthcare Insurance Portability and Accountability Act (HIPAA) of the United States as a Global standard -healthcare associations completely understand the earnestness to coordinate their organizations. Shockingly, the majority of the wellbeing information systems is still restrictive and regularly just serves one particular office inside of a healthcare business. This speaks to a noteworthy deterrent to business reconciliation.

\section{HIS standards \& technologies/ Hospital management}

The developments in technology and internet speed made services like telemedicine a blessing from heaven throughout today's patient care needs. Telemedicine can be alluded to as the procurement of restorative services from a separation. This incorporates analysis, treatment and anticipation of sicknesses. The sorts of telemedicine can be classified as ongoing or prerecorded telemedicine. Likewise the developing technology and differed arrangements in the hospital management area required for the improvement of regular conventions and principles at worldwide level. Such norms and legitimate prerequisites are talked about in further areas.

As per Belgium Federal Public Service-FPS report (2002), high caliber of Data stockpiling, data speed, data trade and systems administration for Hospital Information Systems (HIS) is compulsory for proficient execution of Hospital Information Systems (HIS) [1]. 
Citation: Jamali MC. An Analysis on Transforming Developments in Electronic Hospital Management \& Hospital Information Systems. J Clin Trials Pat 2017;1(1): 3.

ISSN: 2573-3834

Particularly data stockpiling prerequisites of divisions like radiology are exceptionally difficult.

HIPAA privacy guidelines for Medical Records and patient information-One of the key parts of HIPAA rules which need to be consented to by all Hospital management system executing E-Health management systems of worldwide gauges is the privacy rule in HIPAA. The HIPAA Privacy Rule ensures all "individually identifiable wellbeing information" held or transmitted by a secured substance or its business partner, in any structure or media, whether electronic, paper, or oral. The Privacy Rule calls this information "ensured wellbeing information (PHI).

A point by point direction manual on de-distinguishing proof techniques to be received for PHI content in Hospital records and information systems management is given by U.S. Branch of Health \& Human Services [2]. The procedure of de-ID, by which identifiers are expelled from the wellbeing information, mitigates privacy dangers to people also, in this manner backing the auxiliary utilization of data for near adequacy studies, strategy appraisal, life sciences exploration, and different endeavors.

HL7/RIM Development Framework-Health Level Seven (HL7) is one of several American National Standards Institute (ANSI) accredited Standards Developing Organizations, operating in the healthcare arena worldwide. ("Level Seven" refers to the highest level of the International Standards Organization's (ISO) communications model for Open Systems Interconnection (OSI)-the application level). According to Mauro Regio (2005), the HL7 vision is to create an infrastructure for interoperability in the healthcare domain. HL7 uses the reference information model (RIM) to derive domain specific information models and refine them into HL7 message specifications. Traditionally engaged in modeling clinical and administrative data, the most recent version, HL7 version 3.0, is extending its reach to various healthcare business domains such as pharmacy, medical devices, and imaging [3]

\section{E-Hospital management tasks \& capabilities}

To carve out a comprehensive E-Hospital management / Hospital information systems model, some of the various E-Hospital management system tasks are discussed and summarized in the context of the current study.

The most important tasks in hospital information systems can be summarized as follows [4]:

1. Storage and monitoring of patient's condition:

- Accurate and electronically stored medical records of patients (e.g. drug allergies) are provided.

- Visual and auditory warning systems are generated in the event of abnormal test results or other important data.

- Time intervals and / or testing periods for tests on patients to be specified.

- Data Processing and analysis for research oriented and purposes statistical purposes.

2. Management and Data Flow:
- Support automated patient data transfers between departments and institutions.

- Enable graphic or digitized diagnostic images from the hospital database based on the integrated retrieval system.

- Digital signatures, in order to create internal orders electronically.

- Communication by Laboratory Information System.

- Registration of human resources and their properties.

3. Financial Aspects:

- Efficient administration in finances.

- Use and monitoring of medicines and effectivity of the ordering process.

- $\quad$ Expected and actual treatment costs are listed and reported.

- Automated representation of the needs of the nursing staff.

- Status analysis of bed occupancy and overall performance in the hospital information system.

In the public sector domain, some of the successful E-Hospital Management Solutions include that of the, e-Hospital solution by National Informatics Center, India [5]. It is a Hospital Management System that is a workflow based ICT solution for Hospitals specifically meant for the hospitals in Government Sector. This is generic software which includes all major functional areas like laboratory services, patient care, work flow based document/information exchange, human resource and medical records management of a hospital. It is a patient-centric system rather than a series of add-ons to a financial system.

E-Hospital Management Solution (E-HMS) is designed for multispecialty hospitals, to cover a wide range of hospital administration and management processes. E-HMS is an integrated end-to-end Hospital Management System which provides relevant information across the hospital to support hospital administration, effective decision making for patient care and critical financial accounting, in a seamless flow. Some of the salient features of this e-Hospital solution of NIC are included below.

\section{HIS-Success factors}

According to Garrido et al. some of the success factors of HIS deployment are discussed in further sections.

The success of the HIS deployment depends on a number of critical factors includes First, the commitment of senior leadership to implement clear targets and expectations is crucial to the success of the business case [6,7]. Job roles, changes to operational processes and organizational culture will require resources and the strong and consistent support of leadership. All levels of management should be clearly informed and accountable for the key actions that need to be undertaken to maximize system benefits.

Second, timely implementation of the inpatient information system is imperative because the consequent impact of delays on benefits realization is costly. 
Citation: Jamali MC. An Analysis on Transforming Developments in Electronic Hospital Management \& Hospital Information Systems. J Clin Trials Pat 2017;1(1): 3.

Third, because the majority of annual expenses are from labor costs, senior management will need to partner with labor to take advantage of the efficiencies introduced to the workflow by the HIS. Fourth, internal policies must require physicians and frontline staff to comprehensively and accurately codify all hospital discharges and procedures. Finally, workflows must be redesigned to incorporate and exploit the system's functionality.

\section{Conclusion}

The various performance indicators and standards of E-Hospital Management Solution and HIS are discussed in the respective section and cases. Success factors of E-HMS/HIS tend to vary depending upon leadership support, training, technology adoption, user friendliness etc. HIPAA privacy guidelines and HL7/RIM framework are identified as the primary determinants and metrics of Global compliance in developing and implementing successful E-Hospital Management Solutions.
Also the various case study insights on the broader framework of E-Hospital management solution/HIS paves way for future research on Enhancements in E-Hospital Management domain.

\section{References}

1. Bosch VB, Mark Vanautgaerden EB, Bangels M (2002) Recommendations and quality criteria for hospital information systems.

2. Malin B (2010) Guidance on de-identification of protected health information U.S. Department of Health \& Human Services.

3. (2012) HL7 Reference information model, RIM version 2.41. Health Level Seven ${ }^{\circledR}$ International

4. Homayounfar P (2012) Process mining challenges in hospital information systems. Proceedings of the Federated Conference on Computer Science and Information Systems (FEDCSIS), Wroclaw, Poland. pp. 1135-1140.

5. (2013) E-Hospital management solution. National Informatics Center.

6. Garrido T, Raymond B, Jamieson L, Liang L, Wiesenthal A (2004) Making the business case for hospital information systems--a Kaiser permanente investment decision. J Health Care Finance 31: 16-25.

7. Winter A, Brigl B, Wendt T (2003) Modeling hospital information systems. Part 1: The revised three-layer graph-based meta model 3LGM2. Methods Inf Med 42: 544-551. 